\title{
SÉLECTION ET UTILISATION DE L'HABITAT PAR LES JEUNES STADES DE POISSONS D'EAU COURANTE : LE MODĖLE OMBRE COMMUN (THYMALLUS THYMALLUS, L.).
}

\author{
P. SEMPESKI, P. GAUDIN
}

URA CNRS 1974, Ecologie des Eaux Douces et des Grands Fleuves, 43 bd du 11 novembre 1918, 69622 Villeurbanne Cedex, France.

\section{RÉSUMÉ}

L'Ombre commun a servi de modèle biologique dans le cadre de l'étude de la dynamique des changements d'habitats caractérisant les jeunes stades de poissons d'eau courante. Des campagnes d'observations directes, depuis la berge ou en plongée, de jour comme de nuit, associées à des descriptions de l'habitat physique et des prélèvements de poissons et de faune invertébrée benthique et dérivante, ont permis une analyse fine de la sélection et de l'utilisation de l'habitat par les jeunes stades d'ombre et de leur évolution à différentes échelles spatio-temporelles. Différents groupes fonctionnels ont pu être distingués parmi les stades larvaires et juvéniles, en fonction des microhabitats sélectionnés de jour (alimentation) et de nuit (repos), et des différentes stratégies de sélection des proies.

\section{HABITAT SELECTION AND HABITAT USE BY YOUNG STAGES OF RHEOPHILIC FISH SPECIES : THE GRAYLING MODEL (THYMALLUS THYMALLUS, L.).}

\begin{abstract}
The grayling was chosen as a model for the study of habitat shifts dynamics in young stages of rheophilic freshwater fish. Direct observations performed from the river bank or by snorkeling, by day and by night, along with descriptions of physical habitat and sampling of invertebrate fauna, enabled the analysis of habitat selection and habitat use by young stages of grayling and its evolution at different spatial and temporal scales. Different functional groups were distinguished among larval and juvenile stages, depending on habitats selected by day (feeding) or by night (resting) and on feeding strategies.
\end{abstract}

\section{INTRODUCTION}

Chez les poissons, comme chez la plupart des animaux, les jeunes stades représentent une période sensible du cycle vital durant laquelle les risques de mortalité sont particulièrement élevés (LE CREN, 1961 ; ELLIOTT, 1989). Ceci provient du fait que ces jeunes stades ont des capacités limitées, sont peu expérimentés et doivent apprendre à connaître et utiliser leur environnement ; ils sont donc particulièrement vulnérables vis-àvis des pressions externes telles que la compétition, la prédation ou diverses autres perturbations (hydrauliques, physico-chimiques...). De plus, pendant cette période, leur croissance est forte et, par conséquent, leurs capacités d'exploitation du milieu, mais aussi leurs besoins internes changent rapidement. Ainsi, un habitat propice à la croissance et à la survie d'un stade de développement donné peut ne plus correspondre aux besoins du stade suivant. C'est pourquoi, les premiers mois du cycle vital, de nombreuses espèces de poissons sont caractérisées par l'utilisation successive de plusieurs types d'habitats adaptés aux besoins de chaque stade. C'est le cas en particulier chez les poissons rhéophiles, c'est-à-dire inféodés aux milieux lotiques, dont la reproduction se produit généralement dans des zones peu profondes au courant vif, dans des faciès de type radier. Ces caractéristiques physiques semblent favoriser le développement et la survie des oeufs adhérents au substrat ou enfouis sous les graviers, selon les espèces (BALON, 1975 a). 
Cependant, cet environnement devient inadapté une fois les embryons (sensu BALON, 1975 b) éclos ou émergés des graviers (SMITH, 1985), car ceux-ci, comme les alevins (i. e. les individus ayant débuté la phase d'alimentation exogène), ont des capacités de nage limitées (SCOTT, 1985 ; HEGGENES et TRAAEN, 1988). C'est pourquoi l'on rencontre classiquement les jeunes stades d'espèces rhéophiles dans des habitats situés en marge du chenal principal des cours d'eau caractérisés par des vitesses de courant faibles à nulles (MOORE et GREGORY, 1988 ; SCHIEMER et SPINDLER, 1989). En grandissant, les individus acquièrent des capacités de nage supérieures et occupent des habitats plus lotiques.

L'Ombre commun, Thymallus thymallus (L.), est pour plusieurs raisons un modèle biologique de choix pour l'étude de cette dynamique d'utilisation de l'habitat par les jeunes stades de poissons d'eau courante. Sa taille à l'émergence (environ $15 \mathrm{~mm}$ ) et sa localisation le long de la berge au stade larvaire facilitent les repérages et les observations comportementales. Sa croissance élevée et, par suite, l'évolution physiologique et morphologique rapide des individus induisent des changements d'habitats bien marqués entre les différents stades.

\section{SÉLECTION DE L'HABITAT PAR LES JEUNES STADES D'OMBRE}

Après l'émergence des graviers des frayères situées dans des zones de radier au courant vif (FABRICIUS et GUSTAFSON, 1955 ; MÜLLER, 1961 ; GÖNCZI, 1989), les alevins d'ombre se dispersent le long des berges du cours d'eau (SCOTT, 1985; BARDONNET et al., 1991) où ils occupent préférentiellement les zones mortes, petites baies formées par les irrégularités de la berge, caractérisées par de faibles vitesses de courant, généralement $<20 \mathrm{~cm} \mathrm{~s}^{-1}$ (MOORE et GREGORY, 1988 ; SCHIEMER et SPINLDER, 1989 ; VALENTIN et al., 1994). La phase de dispersion post-émergence reste pour l'instant mal connue, on sait seulement que, après un pic d'émergence situé en début de journée, les alevins restent sur les frayères plusieurs heures avant de se disperser à la nuit tombée (BARDONNET et GAUDIN, 1990). Au cours des deux à trois semaines de vie passées dans leur habitat nurserie, les alevins acquièrent progressivement les caractéristiques morphologiques qui feront d'eux des juvéniles aptes à vivre dans le chenal. Des travaux récents ont permis de définir les caractéristiques physiques (vitesse du courant, hauteur d'eau, granulométrie du substrat, forces de cisaillement près du fond) des trois grands types d'habitat successivement occupés par les embryons $(<15 \mathrm{~mm})$, les alevins $(15-30 \mathrm{~mm})$ et les juvéniles d'ombre (> $30 \mathrm{~mm})$, à savoir respectivement les frayères, les zones mortes et certaines zones du chenal. Les différents habitats utilisés sont très caractéristiques (Fig. 1) et certains paramètres physiques comme la vitesse du courant sont fortement sélectionnés dans le milieu (SEMPESKI et GAUDIN, 1995a et b).

\section{DYNAMIQUE JOUR/NUIT D'UTILISATION DE L'HABITAT PAR LES JEUNES STADES D'OMBRE}

Si l'enfouissement des oeufs et des embryons sous plusieurs centimètres de graviers (FABRICIUS et GUSTAFSON, 1955) semble suffire pour maintenir les embryons à l'obscurité (BARDONNET et GAUDIN, 1990) et donc les soustraire aux variations de luminosité liées à l'alternance jour/nuit, cela n'est plus le cas après l'émergence en eau libre. On met alors en évidence un rythme nycthéméral d'utilisation de I'habitat par les jeunes stades d'ombre. SCOTT (1985) démontre ainsi que l'activité alimentaire a lieu principalement de jour, et que son intensité est maximale à l'aube et au crépuscule et minimale au milieu de la nuit. Cette évolution nycthémérale de l'activité alimentaire s'accompagne de déplacements journaliers entre les habitats d'alimentation et de repos (Fig. 2 ; SEMPESKI et GAUDIN, sous presse). A la tombée de la nuit, on observe un mouvement transversal depuis les zones d'alimentation des alevins (la zone morte) et des juvéniles (zone de transition entre zone morte et chenal ou chenal proprement dit) vers les zones de repos près des berges. Les individus sont alors observés, inactifs, posés sur le fond, d'autant plus près de la berge et dans de faibles profondeurs qu'ils sont de petite taille. Les post-émergents inférieurs à $20 \mathrm{~mm}$ sont ainsi couramment repérés contre la berge, dans moins de $1 \mathrm{~cm}$ d'eau. 


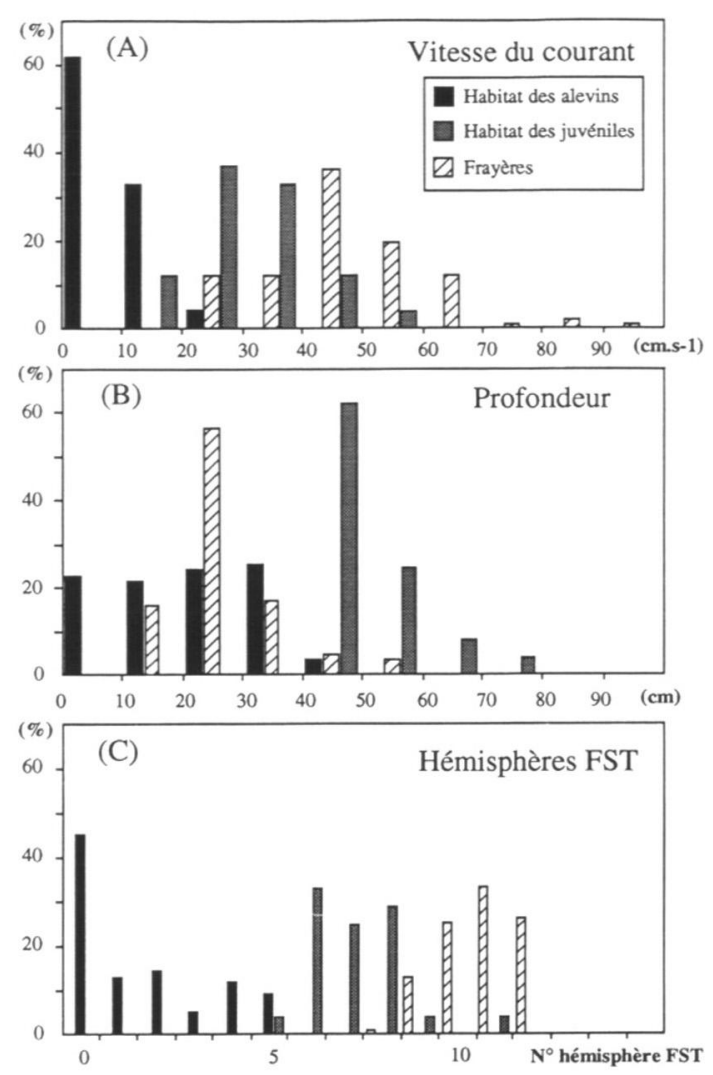

Figure 1 : Vitesse du courant (A), profondeur $(B)$ et numéros d'hémisphères FST (STATZNER \& MÜLLER, 1989) (C) caractérisant les frayères (barres rayées) et les habitats utilisés de jour par les alevins (barres noires) et les juvéniles (barres grises).

Figure 1 : Current velocity (A), water depth (B) and FST-hemisphere numbers (STATZNER \& MUULLER, 1989) (C) characterizing the spawning sites (striped bars) and larval (black bars) and juvenile (grey bars) daytime habitats.

\section{STRATÉGIE ALIMENTAIRE DES JEUNES STADES D'OMBRE}

D'après la littérature, le régime alimentaire des alevins d'ombre apparaît plus ou moins diversifié selon les pays et les types de milieux (rivières, lacs) étudiés (MÜLLER, 1961 ; PERSAT, 1976 ; SCOTT, 1985). Une étude menée sur quatre rivières françaises a montré que le régime alimentaire des ombrets était spécialisé sur les différents stades de chironomides, en particulier les larves et les nymphes, qui représentent généralement plus de $90 \%$ des proies ingérées par les alevins et les juvéniles (SEMPESKI et al., 1995). Seuls quelques taxons (larves d'éphéméroptères, larves de simulies) diversifient le régime des juvéniles. En grandissant, la taille maximale des proies ingérées augmente, en relation avec l'augmentation de la taille de la bouche. Malgré tout, on note une préférence nette pour les proies de $0.5-1 \mathrm{~mm}^{3}$ qui est la classe de biovolume la plus représentée en nombre dans les contenus digestifs (Fig. 3). II est intéressant de noter l'apparition de très grosses proies (jusqu'à $20 \mathrm{~mm}^{3}$ ) dans le régime des juvéniles. L'analyse du comportement alimentaire, par observation directe depuis la berge, met en évidence un changement de stratégie parallèle à l'augmentation en taille des individus et à leur glissement vers le chenal. D'une manière générale, les juvéniles semblent adopter une stratégie minimisant les dépenses énergétiques liées à l'alimentation, en se positionnant légèrement au-dessus du fond (vitesse du courant plus faible que dans la colonne d'eau), en sélectionnant des grosses proies (apport énergétique important), et en retardant leur attaque après repérage d'une proie (réduction de la distance de capture). 


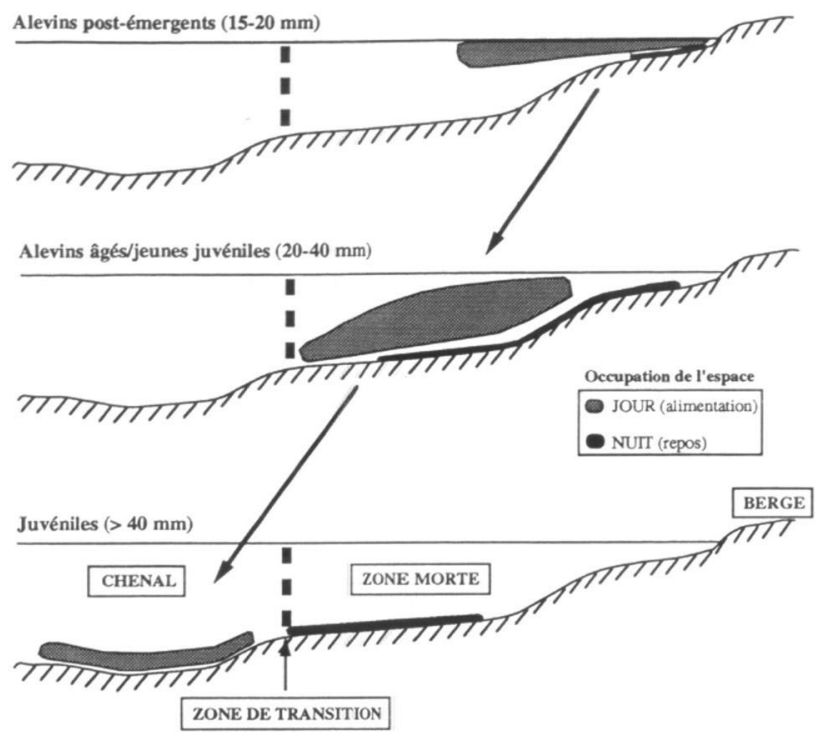

Figure 2 : Dynamique de l'utilisation de l'habitat par les jeunes stades d'ombre. Les différents compartiments de I'habitat utilisés de jour et de nuit sont représentés de manière schématique par des surfaces grisées ou noires, respectivement. Les flèches montrent la tendance des ombrets à glisser vers le chenal en grandissant.

Figure 2 : Dynamic of habitat use in young grayling. The different compartments of the habitat used by day and by night are schematically represented by grey or black surfaces, respectively. Arrows show the tendency of grayling to shift to the river channel with increasing size.

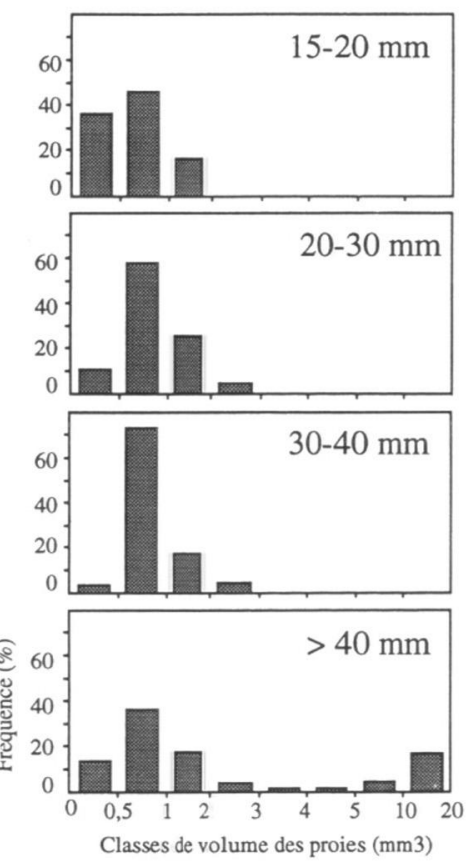

Figure 3 : Importance relative des différentes classes de volume de proies dans l'alimentation d'ombrets de l'Oignin.

Figure 3 : Relative importance of the different classes of volume of prey in the diet of young grayling from the Oignin. 


\section{DISCUSSION}

Il existe chez les jeunes stades d'ombre une dynamique de migration se produisant d'une part à l'échelle saisonnière, entre des habitats spécifiques adaptés aux besoins des différents stades, et d'autre part à l'échelle journalière, entre les habitats d'alimentation et de repos de chaque stade. La stratégie de sélection et d'utilisation de l'habitat apparaît avant tout liée à la taille, et donc aux capacités physiques des individus. Les alevins à l'émergence, aux capacités de nage limitées (HEGGENES et TRAAEN, 1988) s'abritent du courant principal au niveau des zones mortes localisées le long des berges. Ce n'est qu'après quelques semaines et après avoir atteint une taille d'environ $40 \mathrm{~mm}$ que les alevins, devenus des juvéniles, franchissent la limite du courant et rejoignent le chenal (SEMPESKI et GAUDIN, sous presse). Néanmoins, de nuit, les juvéniles reviennent se reposer en zone morte. D'une manière générale, on peut supposer que ces changements d'habitat et de stratégie d'utilisation de l'habitat sont liés à la recherche d'un rendement optimal (WERNER et al., 1974). Ainsi, en se reposant la nuit dans des zones calmes très peu profondes et près des berges, les individus limitent les dépenses énergétiques liées à la nage tout en se protégeant (surtout les plus petits récemment émergés) des prédateurs potentiels tels que les truites adultes. La zone morte représente donc un compartiment du cours d'eau essentiel aux jeunes stades d'ombre, en tant qu'habitat nurserie obligatoire pour les alevins, de jour comme de nuit, et habitat de repos pour les juvéniles la nuit (SEMPESKI et GAUDIN, sous presse). Des expérimentations réalisées en conditions semi-naturelles ont également mis en évidence le rôle de refuge joué par ces zones pour des juvéniles en cas de perturbation hydraulique, telle qu'une simulation d'éclusée (VALENTIN et al., 1994).

Les résultats concernant la sélection de l'habitat et son utilisation tant dans sa dimension physique (stratégie d'occupation de l'espace) que trophique (stratégie alimentaire) par les jeunes ombres ont fait émerger la notion de "groupe fonctionnel", c'està-dire de groupe d'individus homogènes dans leur manière d'utiliser l'habitat (SEMPESKI et GAUDIN, sous presse). Cette notion, qui se distingue de celle de stade de développement, n'est pas comme cette dernière définie a priori sur des critères physiologiques et morphologiques mais est fondée sur des réalités biologiques, c'est là son originalité. D'un point de vue fondamental, cette notion mériterait d'être développée chez d'autres espèces de poissons rhéophiles, salmonidés ou cyprinidés, dont on sait que les jeunes stades utilisent comme l'ombre des habitats latéraux avant de rejoindre le chenal, habitat des adultes (MOORE et GREGORY, 1988 ; SCHIEMER et SPINDLER, 1989 ; HARRIS et al., 1992). Cela permettrait de porter un regard nouveau sur les stratégies d'utilisation de l'habitat chez ces jeunes stades. D'un point de vue plus appliqué, la notion de groupe fonctionnel pourrait remettre en question l'utilisation de courbes de préférences d'habitat établies par stade de développement, dans la mesure où il n'y a pas forcément correspondance entre groupes fonctionnels et stades de développement (SEMPESKI et GAUDIN, sous presse).

\section{REMERCIEMENTS}

Ce travail a été réalisé dans le cadre du Programme Interdisciplinaire de Recherche Environnement (PIRE - CNRS) intitulé "Modélisation de l'utilisation spatiotemporelle de l'habitat par les poissons".

\section{BIBLIOGRAPHIE}

BALON E. K., 1975a. Ecological guilds of fishes : a short summary of the concept and its application. Verh. Internat. Verein. Limnol., 19, 2430-2439.

BALON E. K., 1975b. Terminology of intervals in fish development. J. Fish. Res. Bd. Can., 32, 1663-1670.

BARDONNET A., GAUDIN P., 1990. Influence de la lumière au cours de l'ontogenèse sur l'expression du rythme d'émergence de l'alevin d'Ombre commun, Thymallus thymallus (L. 1758). Bull. Fr. Pêche Piscic., 317, 35-49. 
BARDONNET A., GAUDIN P., PERSAT H., 1991. Microhabitats and diel downstream migration of young grayling (Thymallus thymallus L.). Freshwat. Biol., 26, 365-376.

ELLIOTT J.M., 1989. Mechanisms responsible for population regulation in young migratory trout, Salmo trutta. I. The critical time for survival. J. Anim. Ecol., 58, 987-1001.

FABRICIUS E., GUSTAFSON K.J., 1955. Observations on the spawning behaviour of the grayling, Thymallus thymallus (L.). Rep. Inst. Freshwat. Res. Drottningholm, 36, 75-103.

GÖNCZI A.P., 1989. A study of physical parameters at the spawning sites of the European grayling (Thymallus thymallus L.). Regul. Riv. Res. Manage., 3, 221-224.

HARRIS D.D., HUBERT W.A., WESCHE T.A., 1992. Habitat use by young-of-year brown trout and effects on weighted usable area. Rivers, 3, 99-105.

HEGGENES J., TRAAEN T., 1988. Downstream migration and critical water velocities in stream channels for fry of four salmonid species. J. Fish Biol., 32, 717-727.

LE CREN E.D., 1961. How many fish survive ? Yearbook Riv. Bd Assoc., 76, 57-64.

MOORE K. M.S., GREGORY S.V., 1988. Summer habitat utilization and ecology of cutthroat trout fry (Salmo clarki) in Cascade Mountain streams. Can. J. Fish. Aquat. Sci., 45, 1921-1930.

MÜLLER K., 1961. Die Biologie der Äsche (Thymallus thymallus L.) im Lule Älv (Schwedisch Lappland). Zeit. Fisch. Hilfswissenschaften, 1-3, 173-201.

PERSAT H., 1976. Principaux aspects de l'écologie de l'Ombre commun Thymallus thymallus (L. 1758) (Poissons Salmonidés). Thèse 3ème cycle, Univ. Lyon 1, 69 p.

SCHIEMER F., SPINDLER T., 1989. Endangered fish species of the Danube River in Austria. Regul. Riv. Res. Manage., 4, 397-407.

SCOTT A., 1985. Distribution, growth, and feeding of postemergent grayling Thymallus thymallus in an English river. Trans. Am. Fish. Soc., 114, 525-531.

SEMPESKI P., GAUDIN P., 1995a. Habitat selection by grayling. I. Spawning habitats. J. Fish Biol., 47, 256-265.

SEMPESKI P., GAUDIN P., 1995b. Habitat selection by grayling. II. Preliminary results on larval and juvenile daytime habitats. J. Fish Biol., 47, 345-349.

SEMPESKI P., GAUDIN P., 1995. Size-related changes in diel distribution of young grayling (Thymallus thymallus). Can. J. Fish. Aquat. Sci. (sous presse).

SEMPESKI P., GAUDIN P., PERSAT H., GROLET O., 1995. Diet selection in early-life stages of grayling (Thymallus thymallus). Arch. Hydrobiol., 132, 437-452.

SMITH R. J.F., 1985. The control of fish migration. Ed. Springer Verlag, $243 p$.

STATZNER B., MÜLLER R., 1989. Standard hemispheres as indicators of flow characteristics in lotic benthos research. Freshwat. Biol., 21, 445-459.

VALENTIN S., SEMPESKI P., SOUCHON Y., GAUDIN P., 1994. Short-term habitat use by young grayling (Thymallus thymallus) under variable flow conditions in an experimental stream. Fish. Manage. Ecol., 1, 57-65.

WERNER E.E., MITTELBACH G.G., HALL D.J., GILLIAM J.F., 1974. Experimental tests of optimal habitat use in fish : the role of relative habitat profitability. Ecology, 64, 1525-1539. 